sagt wurde, das Fïllen und Entleeren jedes einzelnen kleinen Ballons! Namentlich wenn es sich um größere Mengen von Säuren handelt, treten die Vorteile des Transportes in Kesselwagen um so deutlicher hervor.

In allerletzter Zeit ist von einer russischen Firma eine neue Art von Kesselwagen konstruiert worden, die in der Hauptsache zur Beförderung von Petroleum, Kerosin und anderen leicht entzündlichen Stoffen dienen soll 1). Der Vorzug dieser neuen Waggons besteht darin, daß sie dank einer sinnreichen Kupplungsvorrichtung bei einem ev. Brande leicht von dem übrigen Zuge losgelöst und entfernt werden können. Außerdem läßt sich bei diesen neuen Wagen ermöglichen, daß mittels einer Hebevorrichtung der Kessel zwischen die Achsen der Fahrzeuge heruntergeklappt wird, wodurch eine, allerdings im Verhältnis zu den anderen Wagen etwas höhere glatte Plattform entsteht. Diese kann zur Verladung von allerhand Waren benutzt werden; es wird anf diese Woise vermieden, daß die Waggons bei entleertem Kessel unbenutzt zurückgehen, wodurch einem gerade auf den in Frage kommenden russischen Eisenbahnstrecken starken Waggonmangel abgeholfen wird.

Es läßt sich aus diesen Ausführungen erkennen, daß, wie unsere Zeit so überaus reich an Fortschritten ist, auch die Mittel zum Transportieren von Flüssigkeiten sich immer mehr vervollkommnet haben. Vor allen Dingen der oben beschriebene Transport von Säuren in mit Hartgummi ausgekleideten Kesselwagen kann nicht genug hervorgehoben werden, weil noch viel zu wenig in der Industrie davon bekannt ist.

Zum Schluß möge noch darauf hingewiesen werden, daB der Bau der Kesselwagen den Bestimmungen der betreffenden Eisenbahnverwaltungen unterliegt. Einige Bestimmungen der preußischen Fisenbahnverwaltung sind in diesen Ausführumgen ja schon erwähnt worden.

\section{Über Salzsäure-Tourills D. R. P. Nr. 106023 \\ und Schleuderapparat zum Reinigen gasförmiger Salzsäure D. R. P. Nr. 183097.}

\section{Von}

Dr. R. Cellakius, Sergiefski-Possad (Rußland)

(Eingeg. den 28.j11. 1907.)

An die Salzsäurebatterie mit Cellarius-Tourills ${ }^{1}$ ) ist neuerdings ein Apparat angegliedert worden, um die Salzsäuregase vom Sulfatofen vor ihrem Eintritt in die Batterie von den mitgeführten Verunreinigungen als Schwefelsäure, Eisen, Arsen zu befreien.

Vor der Beschreibung des Apparates rekapituliere ich kurz das Wesen und den Wert einer Salz-

1) „Petroleum“ 2. Jahrgang Nr. 24,

1) Da die Tourills unter diesem Namen ver. trieben werden, benenne auch ich sie der Kürze halber mit diesem Namen. säurebatterie mit Cellarius-Tourills im Vergleiche mit einer Batterie alten Systems.

Die früher und auch jetzt noch viel üblichen Tourills leiden an mancherlei Fehlern, die einer vollständigen Absorption des HCl-Gases durch Wasser hinderlich sind.

1. Das Verhältnis von Oberfläche zu Flüssigkeitsinhalt des 'Tourills ist sehr ungünstig, infolgedessen wird die durch die Absorption freiwerdende Wärme nur langsam abgegeben.

2. Aus demselben Grunde wird auch eine Wasserkühlung von außen nur geringen Effekt haben, ganz abgesehen davon, daB die Form des Tourills für eine Montage im Kühlkasten sehr ungünstig ist.

3. Beim Durchströmen der sich anreichernden Salzsäure durch die Batterie wird die stärkere Salzsäure von der einlaufenden schwächeren Säure nicht absolut zum nächstfolgenden Tourill verdrängt, sondern es' findet ein ständiges Mischen der starken mit der schwachen Säure statt. Dadurch wird die schwächere Salzsäure schneller, als es prinzipiell wünschenswert ist, zum Anfange, also der SäureabfluBstelle, der Batterie geführt. Durch Versuche wurde ermittelt, daß die zehnfache Wassermenge nötig ist, um eine Säure von $20^{\circ}$ Bé. aus dem Tourill zu verdrängen.

Alle diese Fehler werden beim Cellarius-Tourill eliminiert, und daraus erklärt sich dessen hoher Wirkungswert.

Das Cellarius - Tourill besteht aus zwei konzentrischen miteinander verbundenen Halbzylindern. Die Längsverbindung dient als Boden des Tourills.
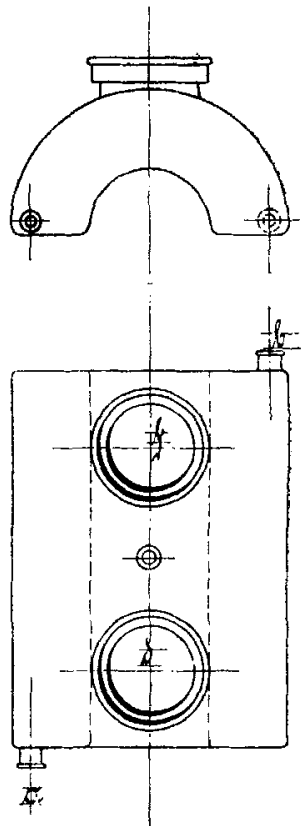

Fig. 1.

Das Tourill wird in zwei Formen ausgeführt. Form I (Fig. 1) wird verwendet, wo keine Wasserkühlung vorgesehen ist, und wo auf Säure, nicht stärker als $22^{\circ}$ Bé,, reflektiert wird.

Die Flüssigkeit tritt durch den Stutzen a dicht über dem Boden ein und verläßt nach dem Uberströmen der Innępwölbung das Tourill auf der gegenüberliegenden Seite direkt über dem Boden durch den Stutzen b.

Form II (Fig. 2) wird bei Wasserkühlung verwendet, und ihm mit können Säuren bis $24^{\circ}$ Bé. erhalten werden. Außerdem kann es wie Form I auch ohne Wasserkühlung montiert werden. Bei ihm liegen dio Stutzen a und $b$ auf derselben Seite des Tourills. Auf der Innenwölbung ist die Leiste c angebracht, die die Rückwand etwa auf $1 / 4$ der Länge des Tourills nicht erreicht. Auf der Außenwölbung sind die zwei üblichen Gasstutzen $d$ und $f$ angebracht. 
Welche Vorteile bietet nun das Cellarius-Tourill gegenüber den alten Tourills ?

1. Das Verhältnis der Oberfläche zum Flüssigkeitsinhalt des Tourills ist um das Mehrfache günstiger.

2. Die Form II läßt sich mit Leichtigkeit im Kühlkasten montieren. Die Flüssigkeitsstutzen werden mittels Gummimanschetten und Flanschen gedichtet und das ganze Tourill bis zu den Gasstutzen ins Wasser gesetzt. Auf diese Weise wird nicht nur die Säure in dünner Schicht von allen Seiten gekühlt, sondern auch das Gas ist gezwungen, stets an wassergekühlten Wänden entlangzustreichen.

Da die Energie der Absorption umgekehrt proportional ist der Höhe der Temperatur von Wasser und Gas, so ist es leicht begreiflich, daß schon da-

Koblenstoffpartikelchen, vermischt mit etwas Teer.

Im allgemeinen wird die Absorption durch diese Schicht nicht erheblich gestört, was aber unbedingt eintritt nach längerem Betrieb und Verbrauch von minderwertigem Rohmaterial.

Müssen nun die Tourills gereinigt werden, so ist dieses bei den üblichen Tourills radikal nur möglich nach Stillegen des Betriebes.

Bei den Cellarius-Tourills jedoch läßt sich dieses jederzeit während des Betriebes bewerkstelligen. Zu dem Zwecke wird schon bei Aufstellung der Batterie in das Verbindungsrohr zweier aufeinanderfolgender Tourills je ein T-Rohr eingesetzt, dessen aufrechtstehender Schenkel etwa die Höhe des Tourills hat (Fig. 3). Diese bietet auch den Vorteil, daß man stets über die Höhe des Flüssigkeitsstandes in jedem Tourill orientiert ist. Durch einfaches Umlegen des T-Rohres können jederzeit im Betriebe die beiden benachbarten Halbtourills entleert und durch event. Nachspülen ohne Mühe gereinigt werden. 7. Die Cellarius-Tourills haben sich, jedenfalls infolge der doppelten Wölbung, wodurch Spannungsdifferenzen leicht ausgeglichen werden, außerordentlich widerstandsfähig gegen Temperatur-

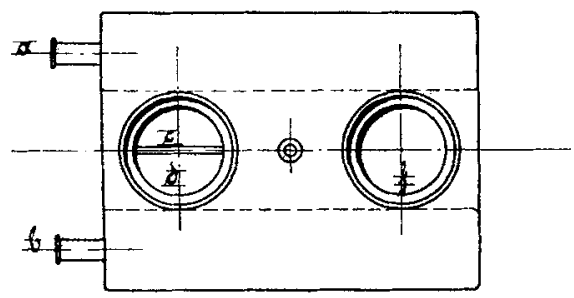

Fig. 2.

durch das wassergekühlte Cellarius-Tourill jedem anderen Tourill weit überlegen ist. Aber noch weitgehender als dieser Vorteil ist, daß

3. durch Anbringen der Flüssigkeitsstutzen am Boden des Gefäßes zu beiden Seiten der Innenwölbung die stärkere Flüssigkeit ohne Vermischen mit schwächerer Säure kontinuierlich zum näcbsten Tourill verdrängt wird.

Durch die $11 /{ }_{2}$ fache Menge Wasser wird Säure von $20^{\circ}$ Bé. vollständig aus dem Tourill verdrängt.

4. Jeder Flüssigkeitsteil muß die Länge und Breite resp. zweimal die Länge des Tourills durchlaufen, durch die Flüssigkeitsschicht einmal aufund einmal absteigen, ehe er zum Abfließen kommt. Uber die Wölbung fließt immer die schwächere Säure und schichtet sich über die stärkere: ein Vermischen ist vollständig ausgeschlossen.

5. Da die Flüssigkeitsstutzen und Verbindungsglasröhren stets unter Flüssigkeit stehen, so ist die Bildung von Luftblasen, die bei den üblichen Tourills, auch solchen mit offenem Ubersteigrohr, oft zu unleidlichen Stauungen führen, ebenfalls absohut ausgeschlossen.

6. Auf der Flüssigkeitsoberfläche in den Tourills bildet sich immer eine schwimmende Schicht, je nach der Qualität des Salzes, der Schwefelsäure (Abfallsäure) des Fettes und der Verkittung mehr oder weniger.

Die Schicht besteht der Hauptsache nach aus verharzten Substanzen und äußerst fein verteilten differenzen erwiesen, so daß ein Bruch, im Betriebe, kommt

8. Durch ihre flache, rechteckige Form nehmen sie bei dem Versand wenig Raum ein, was besonders bei großen Entfernungen sehr in Betracht kommt. Durch die in vorstehendem beschriebenen Vorteile und Eigenschaften des neuen Tourills. das jeder

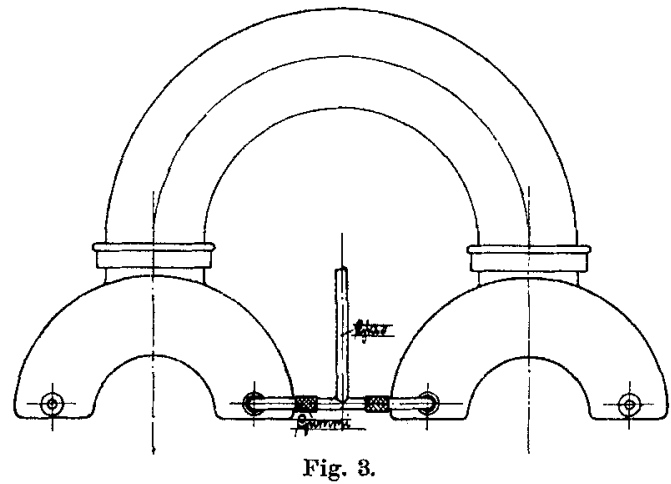

Phase des Absorptionsprozesses angepaßt ist, ist es begreiflich, daß mit ihm Resultate im Salzsäureprozeß gewonnen werden, die bis dahin unerhört waren.

In dreijährigem Betriebe habe ich ein Jahresausbringen von 190-194 Teile technische Salzsäure von $20^{\circ}$ Bé. aus 100 Teilen Salz von $100 \% \mathrm{NaCl}$ erhalten in einer Batterie ohne Wasserkühlung, da für eine Säure von mehr als $22^{\circ}$ Bé. kein Bedürfnis ist. Dabei kann mit solchem Zuge in der Batterie gearbeitet werden, daß bei gleichzeitig geöffneten Arbeitstüren an Muffel und Pfanne kein Gas ausgestoßen wird.

Für eine Tonne Kochsalz sind etwa 20 Gefäße $800 \times 1000 \mathrm{~mm}$ nötig, doch kann bei endstēndigem Plattenturm L u n e - R o h r man die Charge noch um $50 \%$ erhöht werden. 
Das das theoretisch Mögliche überschreitende Ausbringen erklärt sich aus dem $\mathrm{SO}_{3}$-Gehalt der Salzsäure. Jedes $0,1 \% \mathrm{SO}_{3}$ erhöht das Ausbringen um etwa 1,25 , so daß bei einem $\mathrm{SO}_{3}$-Gehalt der Gesamtsäure (Pfannen-Muffel und Waschturmsäure gemischt) von $0,8 \%$ auf die Schwefelsäure ein Ausbringen von 10 kommt; für das $\mathrm{NaCl}$ bleibt dann ein Ausbringen von $193-10=183$. Da aber alle in Literatur und Offerten angegebenen Ausbringungszahlen sich auf technische Salzsäure beziehen, so nehme auch ich keinen Anstand, das Ausbringen mittels der Cellarius-Batterie auf 180-195 Teile technischer Salzsäure von $20^{\circ}$ Bé. aus 100 Teilen $\mathrm{NaCl} 100 \%$ anzugeben, je nach dem geringeren oder größeren Gehalte an $\mathrm{SO}_{3}$. Dieses Ausbringen wird immer erreicht werden, einerlei ob man auf $20-22$ oder 22-24 ige Säure reflektiert, nur muß im letz-

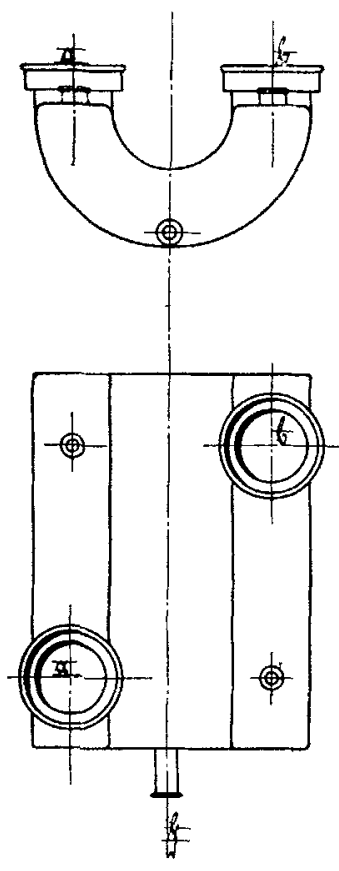

Fig. 4. teren Falle Gasvorkühlung, von der weiter unten die Rede sein wird, und Wasserkühlung der Tourills angewendet werden.

Die Cellarius-Tourills haben sich aber noch in anderer Richtung bewährt und zwar als vorzügliche Gaskühler. Für diesen $\mathrm{Zweck}$ werden sie umgekehrt verwendet, d. h. die beiden Gasstutzen sind auf der Bodenfläche diametral angebracht (Fig. 4), so $\mathrm{da} B$ das Tourill mit der Wölbung nach unten in den Kühlkasten eingesetzt wird. Das bei a eintretende Gas ist gezwungen, zwischen den wassergekühlten Wölbungen durchzustreichen, mit Druck gegen die obere Wölbung, wodurch einestarkeAbkühlung des Gases resp.Kon-

densation der kondensierbaren Bestandteile bewirkt wird. Durch Stutzen f kommen die Kondensationsprodukte zum Ablauf. In dieser Form kommen die Cellarius-Tourills zur Anwendung beim Kühlen der Sulfatgase, beim Salpetersäure- und Anhydridbetriebe, bei Darstellung der wässerigen schwefligen Säure, überhaupt bei jedem Kühlprozesse der chemischen Industrie, wo saure oder alkàlische Stoffe in Betracht kommen.

Ich komme jetzt zur Beschreibung von Einrichtung und Verfahren zur Gewinnung von chemisch reiner Salzsäure direkt beim Sulfatprozesse

Die aus dem Sulfatofen kommenden heißen Gase enthalten außer HCl-Gas und Luft wassergebundene Salzsäure, Schwefelsäure, Eisenchlorür, Arsenchlorür (bei Verwendung As-haltiger Kiese). Um diese Verunreinigungen aus den Gasen vor deren Eintritt in die Batterie auszuscheiden, dient der Schleuderapparat D. R. P. 183097 in seiner Anwendung beim SulfatprozeB. In Fig. 5,1-3 ist die Vorrichtung dargestellt und zwar zeigt:
Fig. 51 einen senkrechten Querschnitt,

Fig. $5_{2}$ Oberteilansicht un ter Weglassung des Lagers,

Fig. $5_{3}$ horizontaler Schnitt nach $A-B$ and Grundriß.

Der aus vier Teilen hergestellte Apparat besteht ans dem Unterteil $a$, dem Mittelteil $b$, dem Oberteil $\mathrm{c}$ und dem Deckel p. Alle Teile sind aufeinander eingeschliffen, $d_{1}$ ist gußeisernes Lager für den Apparat, $d_{2}$ solches für die Spurpfanne.

Der Unterteil ist nach innen gewölbt und zentral durchbohrt zum Durchführen der Achse e des Schleuderflügels $f, g_{1}$ ist Stopfbüchse, h Spurpfanne. Am Unterteil sind die Gaseintrittsstutzen $i_{1}$ und $i_{2}$ angebracht. Auf dem Unterteil steht der Mittelteil b. Dieser besteht aus drei konzentrischen Zylindern $k_{1}, k_{2}$ und $k_{3}$, von denen $k_{2}$ und $k_{3}$ in ihren den Flügeln $f$ gegenüberliegenden Teilen mit Durchlochungen 1 versehen sind. Beide Zylinder sind durch wagerechte durchlochte Querwände $m$ miteinander verbunden. Am AuBenzylinder $k_{1}$ ist der Hahn $n$ angebracht. Auf dem Mittelteil sitzt der Oberteil c auf. An ihm befinden sich die beiden Gasaustrittsstutzen $o_{1}$ und $o_{2}$. Die beiden Innenzylinder sind durch den Deckel $p$ nach außen $a b$. gedichtet. Dieser Deckel hat eine zentrale Durchbohrung für die Achse e der Schleuderflügel und vier seitliche Durchbohrungen für die Rohre $q_{1}-{ }_{4}$, durch die Kondensationsmittel eingelassen werden, $\mathrm{g}_{2}$ ist die Stopfbüchse.

Durch die hohle Welle $r$ des Flügels $f$, welche Teile gleichfalls aus Ton bestehen, ist die eiserne Welle s geführt, die an ihrem äußeren Ende die Antriebsscheibe $\mathbf{t}$ trägt.

Die Wirkungsweise der Vorrichtung ist folgende:

Die zu behandelnden Gase treten durch die Stutzen $i_{1}$ und $i_{2}$ in den Apparat und werden hier zugleich mit dem durch eines der Rohre $\mathrm{q}_{1}-{ }_{4}$ eingelassenen Kondensationsmittel von den sich drehenden Flügeln gegen die Wandung des Zylinders $\mathrm{k}_{3}$ geworfen. Das Gemisch tritt durch die Offnungen 1 des Zylinders $k_{3}$ zwischen die Zylinder $k_{2}$ und $k_{3}$, wo es von dem Kondensat, welches von den Querscheidewänden $\mathrm{m}$ in Tropfen oder feinen Strahlen niederrieselt, getroffen wird. An der Wand des $\mathrm{Zy}$ linders $\mathrm{k}_{2}$ bzw, in deren Durchlochungen 1 findet nochmals eine innige Durchmischung statt, so daß dann die gereinigten aber nicht kondensierten Teile des Gases durch die Stutzen $o_{1}$ und $o_{2}$ aus dem Apparate austreten können. Durch den Apparat können selbstverständlich nur dann kondensierbare Bestandteile ausgeschleudert werden, wenn das Gas vor Eintritt in den Apparat weit unter den Siedepunkt dieser Bestandteile abgekühlt worden ist. Für diesen Zweck wird zwischen dem nicht mit Wasser berieselten Waschturm und dem Schleuderapparat eine Anzahl Gaskïhltourills eingeschaltet, die terrassenförmig übereinander angeordnet sind. Vom Waschturm gehen die Gase zum unteren Kühler und dann nach dem oberen, während das Kühlwasser ihnen entgegenläuft.

Vom oberen Kühler gehen die Gase dann zum Schleuderapparat, wo sie mit der durch $q$ eingelaufenen zerstäubten Flüssigkeit sich mischen und zusammen mit dieser gegen und durch die Lochwände geschleudert werden. Dadurch werden die letzten Spuren der fremden Bestandteile ausge- 
waschen, während die Hauptmenge schon in den Kühlgefäßen ausgeschieden wurde.

Die bei $q$ einlaufende Flüssigkeit ist gesättigte Salzsäure, die immer wieder von neuèm verwendet und nur dann durch frische ersetzt wird, wenn sie allzusehr verunreinigt ist. Sollte bei sehr arsen- dem starken Zuge leicht Teile der zerstäubten Waschflüssigkeit mitgerissen werden, werden die Gase nach dem Verlassen des Schleuderapparates noch durch einen Koksfilter von oben nach unten geführt, der die mechanisch mitgerissenen Teilchen ausscheidet.

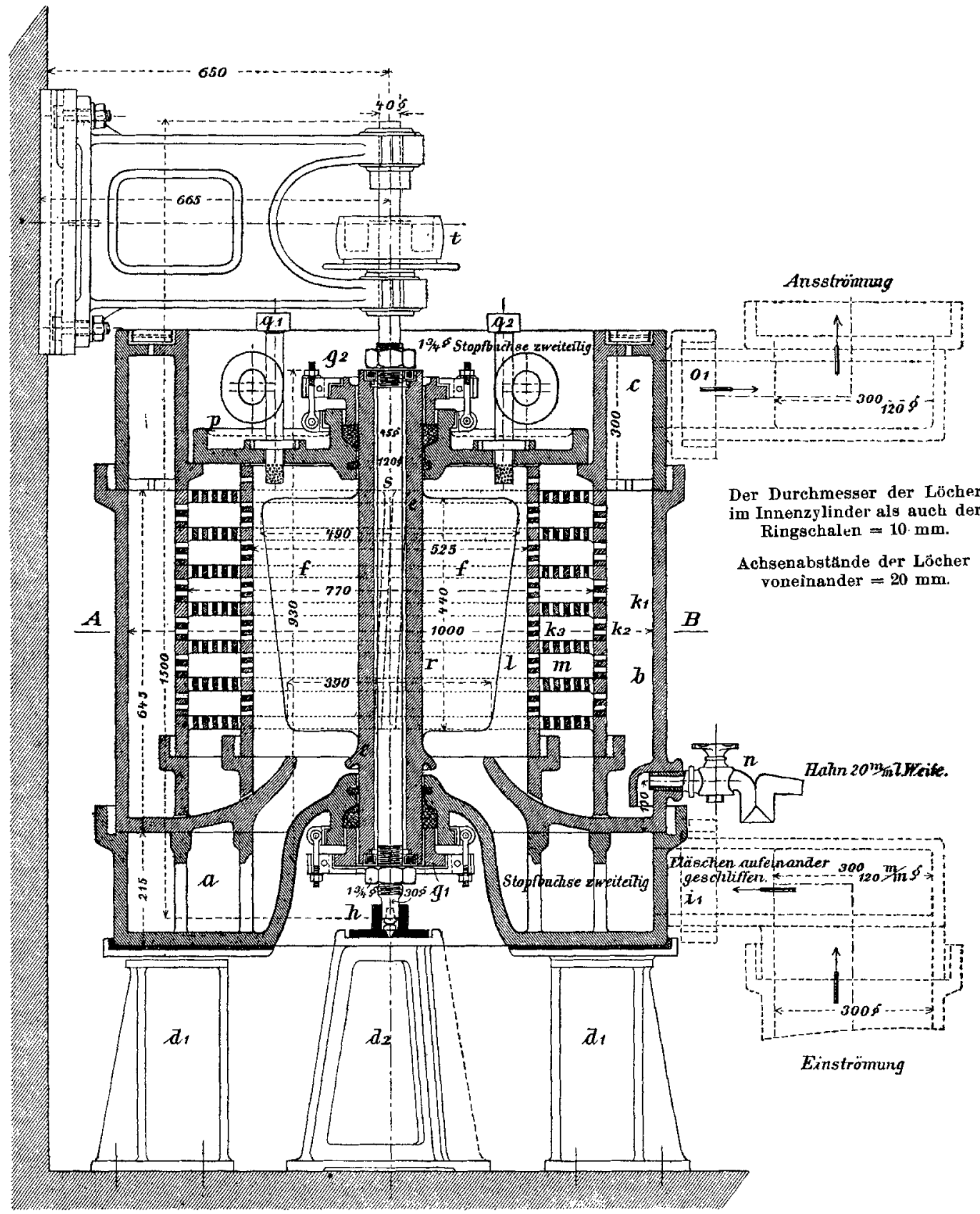

Fig. $\mathbf{b}_{1}$.

haltigen Gasen Arsenchlorïr nicht vollkommen ausgewaschen werden, dann setzt man der Waschflüssigkeit etwas Zinnchlorür zu. Dieses Zinn wird später wiedergewonnen. Die gereinigten $\mathrm{HCl}$-Gase sammeln sich vor dem Außenzylinder $k_{1}$ und gehen durch $o_{1}$ und $o_{2}$ zur Absorptionsbatterie. Da bei
Die Absorptionsbatterie besteht aus wassergekühlten Cellarius-Tourills.

Soll absolut chemisch reine Salzsäure gewonnen werden, dann müssen die Tourills aus eisenfreier Masse, der sogen. „weißen Masse“ der Deutschen Ton- und Steinzeugwerke hergestellt werden, 
und die Batterie muß mit destilliertem Wasser beschickt werden.

In den meisten Fällen wird es sich jedoch darum handeln, überall da, wo bisher gewöbnliche

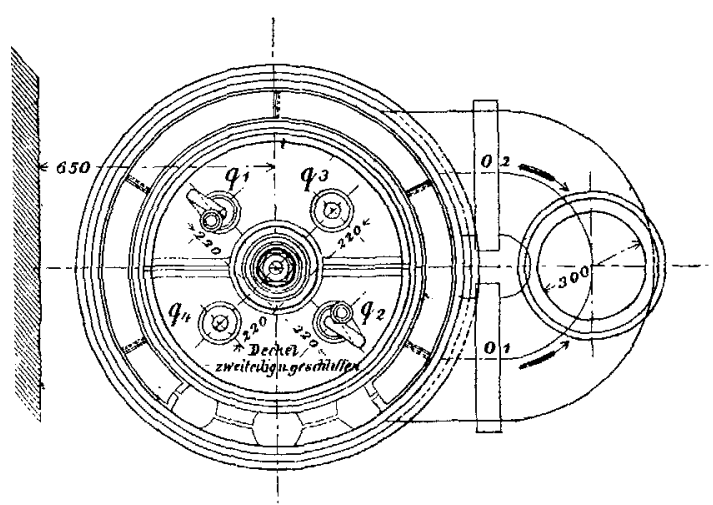

Fig. $5_{2}$.

Salzsäure verwendet wird, weiße, im technischen Sinne von allen Verunreinigungen freie Salzsäure zu präsentieren.

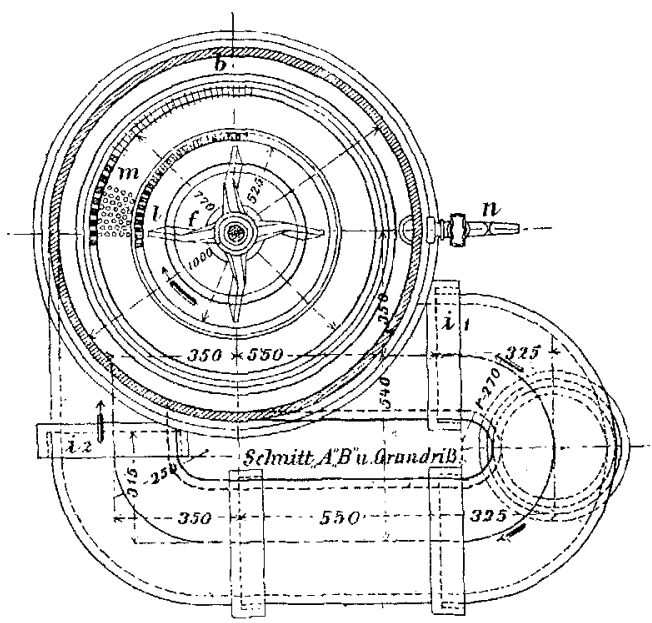

Fig. 5.

Dieses wird erreicht mit enteistem Wasser in den üblichen Cellarius-Tourills.

Zum Schlusse muß ich bemerken, daß die Cellarius-Tourills nach jeder Richtung in der Praxis ausprobiert sind, und die oben angeführte Leistung voll garantiert werden kann, daß dieses aber mit dem Schleuderapparat nicht der Fall ist. Aus äußeren Gründen, auf die ich und die von mir geleitete Fabrik ohne Einfluß sind, konnte der Apparat bisher hier nicht zur Aufstellung kommen.

Ich stelle es daher einem anderen größeren Werke anheim, den Apparat bei sich aufzustellen und als erste auszuprobieren. Ev. Anfragen über Bedingungen und sonst Notwendiges bitte ich entweder direkt an mich oder an die Direktion der Deutschen Ton- und Steinzeugwerke-A.-G. in BerlinCharlottenburg, Berlinerstraße 23, zu richten.

\section{Über den Estrichgips.}

Von Privatdozent Dr. Rohland.

Obwohl d'A ns in seiner Entgegnungl) auf meine Ausführungen ${ }^{2}$ ) über das Thema: „D i e Bildung von Estrichgips im Kolonnenapparat einer Ammoniak s od a f a brik", nur seine falschen Behauptungen wiederholt hat, sehe ich mich doch genötigt, auf zwei von ihm berührte Punkte kurz zurückzukommen.

1. Der Umwandlungspunkt des Gipses in eine anhydridische Modifikation bei $63,5^{\circ}$ kommt bei der Bildung des Estrichipipses g $\mathrm{r} n \mathrm{icht}$ in $\mathrm{Be}$ tracht, bei dieser Temperatur entsteht $k$ e in Estrichgips. Auch van't $H$ of $f$ bemerkt a u $\mathrm{dr} \ddot{\mathrm{ickli}} \mathrm{ch}{ }^{3}$ ), daß die von ihm bei $100^{\circ}$ dargestellte, wasserfreie Modifikation vermutli c h im Estrichgips eine Rolle spielt.

2. Die Beobachtung von $\nabla$ a n't $H$ of $f$, daB bei der Hydratation und Erhärtung des Gipses eine K o n t r a k t i o n stattfindet, bezieht sieh auf den

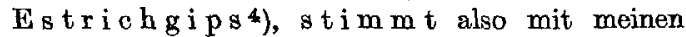
Angaben in meinem Buche ${ }^{5}$ ), sowie in meiner Bearbeitung des Kapitels ,Mörtel“" im A b e g g schen Handbuche ${ }^{6}$ ) volls $t$ ändig ï berein.

Institut für Elektrochemie und technische Chemie der Technischen Hochschule Stuttgart.

1) Diese Z. 20, 1852 (1907)

d) Diese Z. 21, 1228 (1907).

3) Tonindustrieztg. 36, 429 (1902).

4) Der hydraulische oder sogenannte Estrich gips. Sitzungsberichte der Kgl. Pr. Akad. der Wissenschaften 249 (1903).

5) Der Stuck- und Estrichgips. Physikalischchemische Untersuchungen. Quandt $u$. Händel 1904.

6) B. II, Abt. 2.

\section{Referate.}

\section{5. Brenn- und Leuchtstoffe, feste, flïssige und gasförmige; Beleuchtung.}

Bamuel 4. Tucker. Elektrischer Röhrenofen fïr

Temperaturmessungen. (Transact. Amer. Elec-

trochem. Soc., Philadelphia, 2.-8./5. 1907.)

Verf. weist zunächst auf die großen Schwierigkeiten hin, welchen die Bestimmung höherer Tem- peraturen im elektrischen Ofen begegnet. Diese werden hauptsächlich durch die Gegenwart von Dämpfen verursacht, die sich aus den flüchtigen Bestandteilen entwickeln and die Ablesungen am optischen Pyrometer verdunkeln. Zur Beseitigung dieses Mißstandes hat Prof. T u o $\mathrm{k}$ er einen Ofen konstruiert, der nebenstehend in Seitenansicht dargestellt ist. Der Strom geht durch das zu erhitzende Kohlenrohr (A). Am besten für den Zweck geeignet 